\title{
Finite element analysis of diffusion effects on convective heat and the mass transfer of two fluids in a vertical channel
}

\author{
B. Suresh Babu ${ }^{*}$, G. Srinivas ${ }^{2}$ and G.V.P.N. Srikanth ${ }^{3}$ \\ ${ }^{1}$ Sreyas Institute of Engineering and Technology, \\ Hyderabad,Telangana-500 068, India. \\ ${ }^{2}$ Gurunank Institute of Technology, Hyderabad, Telangana, India. \\ ${ }^{3}$ VNR VJIET, Hyderabad. Telangana, India. \\ E-mail:bsureshmaths@gmail.com. \\ Phone:+919963866726
}

\begin{abstract}
In this paper the thermo diffusion and diffusion thermo effects on convective heat and mass transfer along a vertical channel filled with micropolar and viscous fluids have been presented. The coupled system of governing equations is solved numerically using the Galerkin finite element method subjected to the boundary and interface conditions. The effects of the pertinent parameters on the velocity, angular velocity (micro rotation), temperature and diffusion profiles are studied in detail and presented graphically. Furthermore, the rate of heat transfer, mass transfer and shear stress near both walls is entered in tables. It is found that rate of heat transfer enhances vigorously on the hot wall and reduces slowly on the cold wall under the diffusion thermo effect. The rate of mass transfer is higher near the left wall and reduces rapidly on the right wall due to the thermo diffusion effect.
\end{abstract}

Keywords: Micropolar fluid; Viscous fluid; FEM; Magnetic field; Soret and Dufour effects.

\section{INTRODUCTION}

There are many problems in the fields of hydrology and reservoir mechanics in which systems involving two or more immiscible fluids of different densities/viscosities flowing in a channel are encountered. Typical examples of these systems are represented by the air-water, water-salt water, and gas-oil-water systems. The study of the two-phase flow and heat transfer in an inclined channel has been conducted by Malashetty [1, 2]. Malashetty and Leela [3] have analysed the Hartmann flow characteristics of two fluids in a horizontal channel. Muthuraj [4] presented the study of micropolar and viscous fluids in a porous channel using HAM. Navin Kumar and Sandeep Gupta [5] considered the problem of MHD free-convective micropolar and Newtonian fluids in a vertical channel. Srinivas et.al [6] dealt with the flow of two immiscible couple stress fluids between two homogeneous permeable beds.

Micropolar fluids are non-Newtonian fluids with microstructures related to the fluids with a non symmetrical stress tensor. The theory of micropolar fluids has a wide range of applications in measuring the fluid flow in the brain, blood flow in animals, exotic lubricants etc. Micropolar fluids consist of spherical particles suspended in a viscous medium where the deformation can be ignored. Eringen [7] originally formulated 
the theory of the micropolar fluids where the particles allowed to undergo a rotation independent of their linear velocity are taken into account. Additional theoretical concepts and the applications thereof may be found in the books Lukaszewicz [8] and Eringen [9]. The problems of the micropolar fluid flow between two vertical plates (channel) are of great technical interest. A lot of attention has been given by many researchers. Prathap Kumar et al. [10] have studied the analytical solution of the heat transfer of micropolar and viscous fluids. Suresh et al. [11] studied the numerical solution to the heat transfer of micropolar and viscous fluids in a vertical channel. A general non-Newtonian viscous fluid has been considered as an additional fluid in the present study. When heat and mass transfer occur simultaneously in a fluid flow, the relation between the fluxes and driving potentials are more intricate in nature. The energy flux can be generated not only by the temperature gradients but also by the concentration gradients, the flux caused is termed as a diffusion-thermo (Dufour) effect. The mass fluxes caused by the temperature gradient are termed as thermo-diffusion (Soret) effects. In the literature, a large number of studies dealing with the effects of the Dufour and Soret parameters on heat and mass transfer problems on Newtonian and viscoelastic fluids have appeared $[12,13]$. These effects are considered as second order phenomena and have often been neglected in the heat and mass transfer processes [14-16]. Bég et al. [17] emphasised the effect of Soret and Dufour on convective heat and mass transfer. The temperature and concentration gradients leading to the Soret and Dufour effects exert a greater impact on the flow, heat and mass transfer in a two fluid passage than in a single fluid passage.

MHD is the science concerned with the motions of electro fluids and their interactions with magnetic fields. It is a vital branch and it is comparatively new in the field of fluid dynamics. Lohsasbi and Sahai [18] studied the two-phase MHD flow and heat transfer in a parallel plate channel with the fluid in one phase being electrically conducting. Siva Reddy et al. [19] created a numerical model to analyse the heat and mass transfer effects on the MHD natural convection flow past an impulsively moving perpendicular sheet with ramped temperature. Stamenkovic et al. [20] examined the MHD flow of two immiscible and electrically conducting fluids within isothermal, insulated moving sheets under an applied electrical and inclined magnetic effect. Satya Narayana [21] investigated the heat and mass transfer along a vertical porous plate under the combined buoyancy force effects of thermal and species diffusion in the presence of a transversely applied uniform magnetic field, while also taking into account the hall currents. The dynamics of the steady, two-dimensional magnetohydrodynamics (MHD) free convective flow of micropolar fluids along a vertical porous surface embedded in a thermally stratified medium are investigated by Koriko et al. [22]. Keeping in view the wide area of practical importance of multi fluid flows and of the Soret and Dufour effects as mentioned above, the novelty of this study is to investigate the thermo-diffusion and diffusion-thermo effects on convective heat and the mass transfer of micropolar and viscous fluids in a vertical channel using the Galerkin finite element method with inclusion of the physical forces of viscous dissipation and of the magnetic field.

\section{METHODS AND MATERIALS}

\section{Mathematical Formulation}

The two infinite parallel plates are placed at $Y=-h_{1}$ and $Y=h_{2}$ along the Y-direction initially as shown in Figure 1 and both plates are isothermal with different temperatures $T_{1}$ and $T_{2}$, respectively. The distance $-h_{1} \leq Y \leq 0$ represents region-1 and the distance $0 \leq Y \leq h_{2}$ represents region-2 where the first region is filled with micropolar fluid and 
the second region is filled with viscous fluid. The fluid flow in the channel is due to buoyancy forces.

In order to develop the governing equations for the problem considered, the following assumptions are made:

1. The flow is assumed to be one-dimensional, steady, laminar, immiscible and incompressible.

2. The transport properties of both fluids are assumed to be constant.

3. The fluid flow in the channel is due to buoyancy forces.

4. The fluid flow is fully developed.

5. The flow, temperature and species concentration are assumed to be continuous at the interface.

6. Each of the walls are isothermal and have a constant species concentration and $T_{1}>T_{2}, C_{1}>C_{2}$.

7. The flow is assumed to follow the Boussinesq approximation.

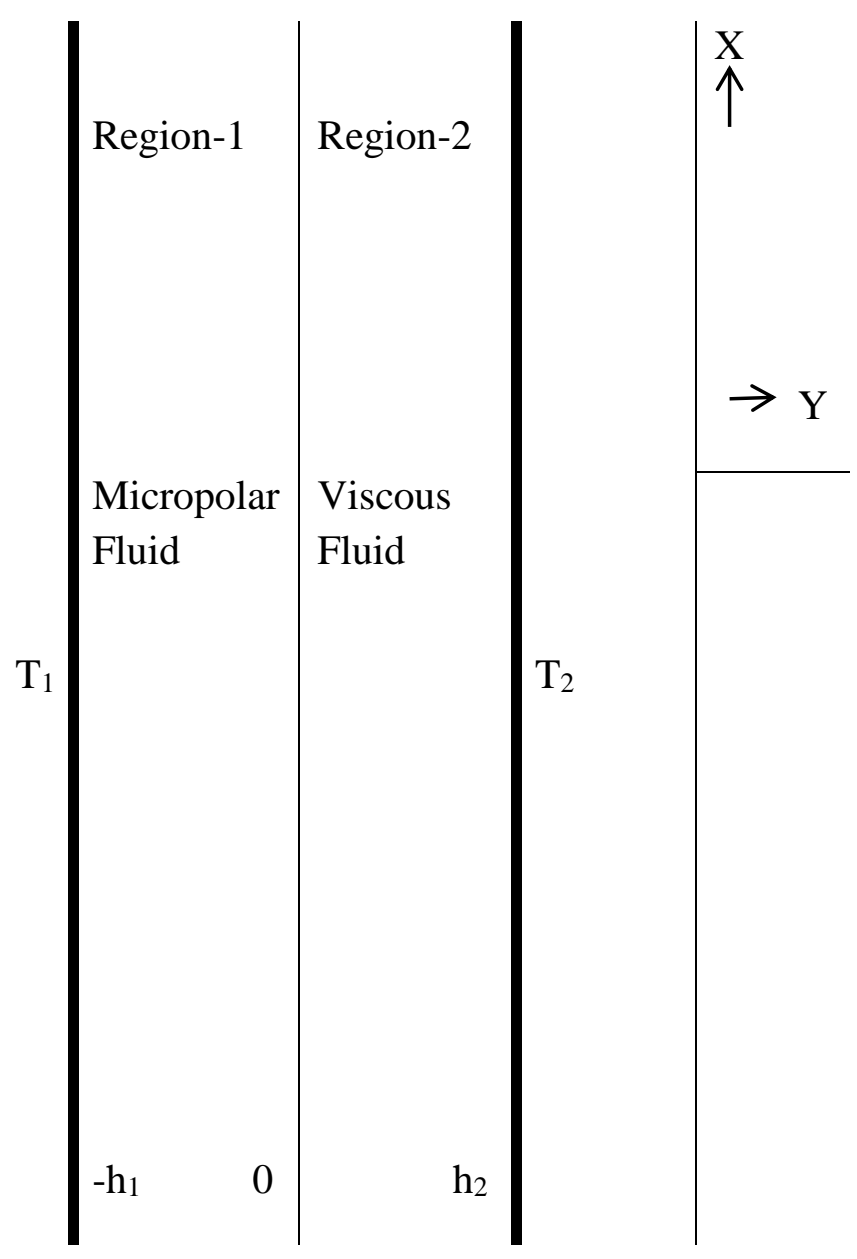

Figure 1. Geometry of the problem. 


\section{Governing Equations}

Region-1:

$$
\begin{gathered}
\frac{d U_{1}}{d Y}=0 \\
\rho_{1}=\rho_{0}\left[1-\beta_{1 T}\left(T_{1}-T_{0}\right)-\beta_{1 C}\left(\mathrm{C}_{1}-C_{0}\right)\right][\text { State] } \\
U_{1} \frac{d U_{1}}{d Y}-\frac{\mu_{1}+K}{\rho_{1}} \frac{d^{2} U_{1}}{d Y^{2}}-\frac{K}{\rho_{1}} \frac{d n}{d Y}-g \beta_{1 T}\left(T_{1}-T_{0}\right)-g \beta_{1 C}\left(C_{1}-C_{0}\right)+\frac{\sigma B_{0}{ }^{2} U_{1}}{\rho_{1}}=0
\end{gathered}
$$

[Momentum]

$$
\gamma \frac{d^{2} n}{d Y^{2}}-K\left[2 n+\frac{d U_{1}}{d Y}\right]=0 \quad \text { [Conservation of the Angular Momentum] }
$$

where $\gamma=\left(\mu_{1}+\frac{K}{2}\right) j$

$$
\begin{aligned}
& \rho_{1} C_{p} U_{1} \frac{d T_{1}}{d Y}- k_{1} \frac{d^{2} T_{1}}{d Y^{2}}-\mu_{1}\left(\frac{d U_{1}}{d Y}\right)^{2}-\frac{\rho_{1} D_{1} K_{T 1}}{C_{S 1}} \frac{d^{2} C_{1}}{d Y^{2}}=0 \\
& U_{1} \frac{d C_{1}}{d Y}-D_{1} \frac{d^{2} C_{1}}{d Y^{2}}-\frac{D_{1} K_{T 1}}{T_{M}} \frac{d^{2} T_{1}}{d Y^{2}}=0 \text { [Diffusion] }
\end{aligned}
$$

Region-2:

$$
\begin{aligned}
& \frac{d U_{2}}{d Y}=0 \\
& \rho_{2}=\rho_{0}\left[1-\beta_{2 T}\left(T_{2}-T_{0}\right)-\beta_{2 C}\left(\mathrm{C}_{2}-C_{0}\right)\right] \\
& U_{2} \frac{d U_{2}}{d Y}-\frac{\mu_{2}}{\rho_{2}} \frac{d^{2} U_{2}}{d Y^{2}}-g \beta_{2 T}\left(T_{2}-T_{0}\right)-g \beta_{2 C}\left(C_{2}-C_{0}\right)+\frac{\sigma B_{0}{ }^{2} U_{2}}{\rho_{2}}=0 \text { [Momentum] (9) } \\
& \rho_{2} C_{p} U_{2} \frac{d T_{2}}{d Y}-k_{2} \frac{d^{2} T_{2}}{d Y^{2}}-\mu_{2}\left(\frac{d U_{2}}{d Y}\right)^{2}-\frac{\rho_{2} D_{2} K_{T 2}}{C_{S 2}} \frac{d^{2} C_{2}}{d Y^{2}}=0 \quad \text { [Energy] } \\
& U_{2} \frac{d C_{2}}{d Y}-D_{2} \frac{d^{2} C_{2}}{d Y^{2}}-\frac{D_{2} K_{T 2}}{T_{M}} \frac{d^{2} T_{2}}{d Y^{2}}=0
\end{aligned}
$$

To solve the above system of Eqs. (1) to (11), we considered the following boundary and interface conditions proposed by Ariman [23].

$$
\begin{aligned}
& U_{1}=0 \text { at } Y=-h_{1}, U_{2}=0 \text { at } Y=h_{2}, U_{1}(0)=U_{2}(0), \\
& T=T_{1} \text { at } Y=-h_{1}, T=T_{2} \text { at } Y=h_{2}, T_{1}(0)=T_{2}(0), \\
& C=C_{1} \text { at } Y=-h_{1}, C=C_{2} \text { at } Y=h_{2}, C_{1}(0)=C_{2}(0), \\
& n=0 \text { at } Y=-h_{1},\left(\mu_{1}+K\right) \frac{d U_{1}}{d Y}+K n=\mu_{2} \frac{d U_{2}}{d Y} \text { at } Y=0, \\
& \frac{d n}{d Y}=0 \text { at } Y=0, k_{1} \frac{d T_{1}}{d Y}=k_{2} \frac{d T_{2}}{d Y} \text { at } Y=0, D_{1} \frac{d C_{1}}{d Y}=D_{2} \frac{d C_{2}}{d Y} \text { at } Y=0 .
\end{aligned}
$$


The following variables are used to render the system of Eqs. (1) to (11) into a dimensionless form:

$$
\begin{aligned}
& y_{1}=\frac{Y_{1}}{h_{1}}, y_{2}=\frac{Y_{2}}{h_{2}}, u_{1}=\frac{U_{1}}{U_{0}}, u_{2}=\frac{U_{2}}{U_{0}}, \theta_{1}=\frac{T_{1}-T_{0}}{\Delta T}, \theta_{2}=\frac{T_{2}-T_{0}}{\Delta T}, N=\frac{h_{1}}{U_{0}} n, K^{\prime}=\frac{\mu_{1}}{K}, \\
& c_{1}=\frac{C_{1}-C_{0}}{\Delta C}, c_{2}=\frac{C_{2}-C_{0}}{\Delta T}, C_{S}=\frac{C_{S 1}}{C_{S 2}}, K_{T}=\frac{K_{T 1}}{K_{T 2}}, D=\frac{D_{1}}{D_{2}}, h=\frac{h_{1}}{h_{2}}, m=\frac{\mu_{1}}{\mu_{2}}, \alpha=\frac{k_{1}}{k_{2}}, \\
& \rho=\frac{\rho_{1}}{\rho_{2}}, b=\frac{\beta_{1 T}}{\beta_{2 T}}, v=\frac{v_{1}}{v_{2}}, \\
& G r=\frac{g \beta_{1 T} \Delta T h_{1}^{3}}{v_{1}{ }^{2}} \text { (Grashof number), } R=\frac{U_{0} h_{1}}{v_{1}} \text { (Reynolds number), } \\
& S r=\frac{D_{1} K_{T 1} \Delta T_{1}}{T_{M} \Delta C_{1} U_{0} h} \text { (Soret number), } S_{c}=\frac{D_{1}}{v_{1}}(\text { Schmidt number), } \\
& D_{u}=\frac{D_{1} K_{T 1} \Delta C}{C_{P} C_{S 1} v_{1} \Delta T} \text { (Dufour number), } M=\frac{\sigma B_{0}{ }^{2} h_{1}{ }^{2}}{\mu_{1}} \text { (Magnetic field parameter), } \\
& \operatorname{Pr}=\frac{\mu_{1} C p}{k_{1}} \text { (Prandtl number), Ec }=\frac{U_{0}{ }^{2}}{C p \Delta T} \text { (Eckert number). }
\end{aligned}
$$

The dimensionless form of the governing equations thus obtained is:

Region-1:

$$
\begin{gathered}
\frac{d^{2} N}{d y^{2}}-\frac{2 K^{\prime}}{2+K^{\prime}}\left(2 N+\frac{d u_{1}}{d y}\right)=0 \\
R u_{1} \frac{d \mathrm{u}_{1}}{d y}-\left(1+\frac{1}{K^{\prime}}\right) \frac{d^{2} u_{1}}{d y^{2}}-\frac{1}{K^{\prime}} \frac{d N}{d y}-\frac{G r}{R} \theta_{1}-\frac{G c}{R} c_{1}+M u_{1}=0 \\
u_{1} \frac{d \theta_{1}}{d y}-\frac{1}{\operatorname{Pr} R} \frac{d^{2} \theta_{1}}{d y^{2}}-\frac{E c}{R}\left(\frac{d u_{1}}{d y}\right)^{2}-\frac{D_{u}}{R} \frac{d^{2} c_{1}}{d y^{2}}=0 \\
u_{1} \frac{d c_{1}}{d y}-\frac{1}{S_{c} R} \frac{d^{2} c_{1}}{d y^{2}}-S_{r} \frac{d^{2} \theta_{1}}{d y^{2}}=0
\end{gathered}
$$

Region -2

$$
\begin{aligned}
& R u_{2} \frac{d \mathrm{u}_{2}}{d y}-\frac{d^{2} u_{2}}{d y^{2}}-\frac{m}{b \rho h^{2}} \frac{G r}{R} \theta_{2}-\frac{m}{b \rho h^{2}} \frac{G c}{R} c_{2}+\frac{M \mu}{h^{2}} u_{2}=0 \\
& u_{2} \frac{d \theta_{2}}{d y}-\frac{\rho h}{\alpha} \frac{1}{\operatorname{Pr} R} \frac{d^{2} \theta_{2}}{d y^{2}}-\frac{\rho h}{m} \frac{E c}{R}\left(\frac{d u_{2}}{d y}\right)^{2}-\frac{c_{s} h}{D K_{T}} \frac{D_{u}}{R} \frac{d^{2} c_{2}}{d y^{2}}=0 \\
& u_{2} \frac{d c_{2}}{d y}-\frac{h}{D}\left(\frac{1}{S c R}\right) \frac{d^{2} c_{2}}{d y^{2}}-\frac{h}{K_{T} D} S r \frac{d^{2} \theta_{2}}{d y^{2}}=0
\end{aligned}
$$

The dimensionless boundary and interface conditions thus formed are:

$$
\begin{aligned}
& u_{1}=0 \text { at } y=-1, u_{2}=0 \text { at } y=1, u_{1}(0)=u_{2}(0), \\
& \theta_{1}=1 \text { at } y=-1, \theta_{2}=0 \text { at } y=1, \theta_{1}(0)=\theta_{2}(0),
\end{aligned}
$$




$$
\begin{gathered}
c_{1}=1 \text { at } y=-1, c_{2}=0 \text { at } y=1, c_{1}(0)=c_{2}(0), \\
N=0 \text { at } y=-1, \frac{d u_{1}}{d y}+\frac{K^{\prime}}{1+K^{\prime}} N=\frac{1}{m h\left(1+K^{\prime}\right)} \frac{d u_{2}}{d y} \text { at } y=0, \\
\frac{d N}{d y}=0 \text { at } y=0, \frac{d \theta_{1}}{d y}=\frac{1}{h \alpha} \frac{d \theta_{2}}{d y} \text { at } y=0, \frac{d c_{1}}{d y}=\frac{1}{h D} \frac{d c_{2}}{d y} \text { at } y=0 .
\end{gathered}
$$

\section{Solution of the Problem}

The dimensionless coupled differential equations generated by the fluid flows are solved numerically using the regular Galerkin Finite Element method as given by J.N. Reddy [24] and Zienkiewicz [25]. For the problem discussed here, it is considered that one dimensional region is divided into 100 linear elements and each element is 3 nodded. The element equations associated with the Eqs. (12) to (18) are derived as given by Sedighi et al. [26]:

$$
\begin{gathered}
\int_{y_{i}}^{y_{i+1}}\left(\frac{d^{2} N}{d y^{2}}-\frac{2 K^{\prime}}{2+K^{\prime}}\left(2 N+\frac{d u_{1}}{d y}\right)\right) \eta_{k} d y=0 \\
\int_{y_{i}}^{y_{i+1}}\left(R u_{1} \frac{d \mathrm{u}_{1}}{d y}-\left(1+\frac{1}{K^{\prime}}\right) \frac{d^{2} u_{1}}{d y^{2}}-\frac{1}{K^{\prime}} \frac{d N}{d y}-\frac{G r}{R} \theta_{1}-\frac{G c}{R} c_{1}+M u_{1}\right) \eta_{k} d y=0 \\
\int_{y_{i}}^{y_{i+1}}\left(u_{1} \frac{d \theta_{1}}{d y}-\frac{1}{\operatorname{Pr} R} \frac{d^{2} \theta_{1}}{d y^{2}}-\frac{E c}{R}\left(\frac{d u_{1}}{d y}\right)^{2}-\frac{D_{u}}{R} \frac{d^{2} c_{1}}{d y^{2}}\right) \eta_{k} d y=0 \\
\int_{y_{i}}^{y_{i+1}}\left(u_{1} \frac{d c_{1}}{d y}-\frac{1}{S_{c} R} \frac{d^{2} c_{1}}{d y^{2}}-S_{r} \frac{d^{2} \theta_{1}}{d y^{2}}\right) \eta_{k} d y=0
\end{gathered}
$$

Region -2

$$
\begin{aligned}
& \int_{y_{i}}^{y_{i+1}}\left(R u_{2} \frac{d \mathrm{u}_{2}}{d y}-\frac{d^{2} u_{2}}{d y^{2}}-\frac{m}{b \rho h^{2}} \frac{G r}{R} \theta_{2}-\frac{m}{b \rho h^{2}} \frac{G c}{R} c_{2}+\frac{M \mu}{h^{2}} u_{2}\right) \chi_{k} d y=0 \\
& \int_{y_{i}}^{y_{i+1}}\left(u_{2} \frac{d \theta_{2}}{d y}-\frac{\rho h}{\alpha} \frac{1}{\operatorname{Pr} R} \frac{d^{2} \theta_{2}}{d y^{2}}-\frac{\rho h}{m} \frac{E c}{R}\left(\frac{d u_{2}}{d y}\right)^{2}-\frac{c_{s} h}{D K_{T}} \frac{D_{u}}{R} \frac{d^{2} c_{2}}{d y^{2}}\right) \chi_{k} d y=0 \\
& \int_{y_{i}}^{y_{i}}\left(u_{2} \frac{d c_{2}}{d y}-\frac{h}{D}\left(\frac{1}{S c R}\right) \frac{d^{2} c_{2}}{d y^{2}}-\frac{h}{K_{T} D} S r \frac{d^{2} \theta_{2}}{d y^{2}}\right) \chi_{k} d y=0
\end{aligned}
$$

where $\eta_{k}$ and $\chi_{k}$ are the shape functions of a typical element $\left(y_{i}, y_{i+1}\right)$ in region 1 and 2 , respectively. The Langrange interpolation polynomials are used as the shape functions at each of the nodes: 


$$
\begin{gathered}
\eta_{i}^{1}=\frac{\left(y-\left(\frac{2 i-101}{100}\right)\right)\left(y-\left(\frac{2 i-100}{100}\right)\right)}{\left(\left(\frac{2 i-102}{100}\right)-\left(\frac{2 i-101}{100}\right)\right)\left(\left(\frac{2 i-102}{100}\right)-\left(\frac{2 i-100}{100}\right)\right)}, \\
\eta_{i}^{2}=\frac{\left(y-\left(\frac{2 i-102}{100}\right)\right)\left(y-\left(\frac{2 i-100}{100}\right)\right)}{\left(\left(\frac{2 i-101}{100}\right)-\left(\frac{2 i-102}{100}\right)\right)\left(\left(\frac{2 i-101}{100}\right)-\left(\frac{2 i-100}{100}\right)\right)}, \\
\eta_{i}^{3}=\frac{\left(y-\left(\frac{2 i-101}{100}\right)\right)\left(y-\left(\frac{2 i-102}{100}\right)\right)}{\left(\left(\frac{2 i-100}{100}\right)-\left(\frac{2 i-102}{100}\right)\right)\left(\left(\frac{2 i-100}{100}\right)-\left(\frac{2 i-101}{100}\right)\right)}
\end{gathered}
$$

and similarly for $\chi_{i}^{1}, \chi_{i}^{2}, \chi_{i}^{3}$.

On integrating the above equations and by replacing the finite element Galerkin approximations given as

$$
\begin{gathered}
u_{1}^{i}=\sum_{j=1}^{3} u_{j}^{i} \eta_{j}^{i}, c_{1}^{i}=\sum_{j=1}^{3} c_{j}^{i} \eta_{j}^{i}, N^{i}=\sum_{j=1}^{3} N_{j}^{i} \eta_{j}^{i} \\
\theta_{1}^{i}=\sum_{j=1}^{3} \theta_{j}^{i} \eta_{j}^{i}, u_{2}^{i}=\sum_{j=1}^{3} u_{j}^{i} \chi_{j}^{i}, c_{2}^{i}=\sum_{j=1}^{3} c_{j}^{i} \chi_{j}^{i}, \theta_{2}^{i}=\sum_{j=1}^{3} \theta_{j}^{i} \chi_{j}^{i} .
\end{gathered}
$$

From Eq. (20) we get

$$
\int_{\mathrm{y}_{i}}^{\mathrm{y}_{\mathrm{i}+1}} \frac{\mathrm{dN}^{i}}{\mathrm{dy}} \frac{\mathrm{d} \eta_{k}}{\mathrm{dy}} \mathrm{dy}+\frac{2 \mathrm{~K}^{\prime}}{2+\mathrm{K}^{\prime}} \int_{\mathrm{y}_{i}}^{\mathrm{y}_{\mathrm{i}+1}}\left[2 \mathrm{~N}^{i} \eta_{k} \mathrm{dy}-\frac{\mathrm{d} \eta_{k}}{\mathrm{dy}} u_{1}^{i}\right] d y=\left[\eta_{k} \frac{\mathrm{dN}^{i}}{\mathrm{dy}}+\eta_{k} u_{1}^{i}\right]_{\mathrm{y}_{i}}^{\mathrm{y}_{\mathrm{i}+1}}
$$

The stiffness matrix equation corresponding to it is,

$$
\left[a_{k j}^{i}\right]\left[N_{k}^{i}\right]+\left[b_{k j}^{i}\right]\left[u_{k}^{i}\right]=\left[Q_{1 j}^{i}\right]
$$

where $a_{k j}^{i}=\int_{\mathrm{y}_{i}}^{\mathrm{y}_{\mathrm{i}+1}} \frac{\mathrm{d} \eta_{k}}{\mathrm{dy}} \frac{\mathrm{d} \eta_{j}^{i}}{\mathrm{dy}} \mathrm{dy}+\frac{2 \mathrm{~K}^{\prime}}{2+\mathrm{K}^{\prime}} \int_{\mathrm{y}_{i}}^{\mathrm{y}_{\mathrm{i}+1}}\left[2 \eta_{k} \eta_{j}^{i}\right] d y$

$$
b_{k j}^{i}=-\frac{2 \mathrm{~K}^{\prime}}{2+\mathrm{K}^{\prime}} \int_{\mathrm{y}_{i}}^{\mathrm{y}_{\mathrm{i}+1}}\left[\frac{\mathrm{d} \eta_{j}^{i}}{\mathrm{dy}} \eta_{k}\right] d y \text { and } Q_{1 j}^{i}=\left[\eta_{k} \frac{\mathrm{dN}^{i}}{\mathrm{dy}}+\eta_{k} u_{1}^{i}\right]_{\mathrm{y}_{i}}^{\mathrm{y}_{\mathrm{i}+1}}
$$

Similarly, we get the stiffness matrix equations corresponding to Eqs. (21) to (26). All these matrix equations are solved iteratively until the desired accuracy of $10^{-5}$ is attained using the Mathematica 10.4 package. The convergence of the method is validated 
with the attainment of the boundary conditions and the numerical solution obtained by this method is compared with the analytical approach of Prathap Kumar et al. [10].

For practical engineering applications, the quantities of the Nusselt number, shear stress and Sherwood numbers are calculated at both walls by using the expressions:

$$
\begin{gathered}
N u_{1}=\left[\frac{\partial \theta_{1}}{\partial y}\right]_{y=-1}, N u_{2}=\left[\frac{\partial \theta_{2}}{\partial y}\right]_{y=1}, S c_{1}=\left[\frac{\partial c_{1}}{\partial y}\right]_{y=-1}, S c_{2}=\left[\frac{\partial c_{2}}{\partial y}\right]_{y=1}, S t_{1}=\left[\frac{\partial u_{1}}{\partial y}\right]_{y=-1}, \\
S t_{2}=\left[\frac{\partial u_{2}}{\partial y}\right]_{y=1} .
\end{gathered}
$$

\section{RESULTS AND DISCUSSION}

The numerical solution of the system of equations is analysed for different values of the governing parameters and the results are presented graphically. The Grashof number (Gr), Mass Grashof number (Gc), Reynolds number (R), Magnetic field parameter (M), Material parameter (K'), Dufour number (Du), Schmidt number $(\mathrm{Sc})$, Soret number $(\mathrm{Sr})$, Eckert number $(\mathrm{Ec})$ are fixed as $\mathrm{Gr}=5, \mathrm{Gc}=5, \mathrm{R}=3, \mathrm{M}=3, \mathrm{~K}^{\prime}=0.1, \mathrm{Du}=0.08, \mathrm{Sr}=0.1$, $\mathrm{Sc}=0.66, \mathrm{Sr}=0.001$ for all the profiles with the exception of the varying parameter. All our results are validated with Prathap Kumar et al. [10] and an illustration of the effect of the ratio of $\mathrm{Gr}$ and $\mathrm{R}$ on the velocity profiles is shown in Figure 2.

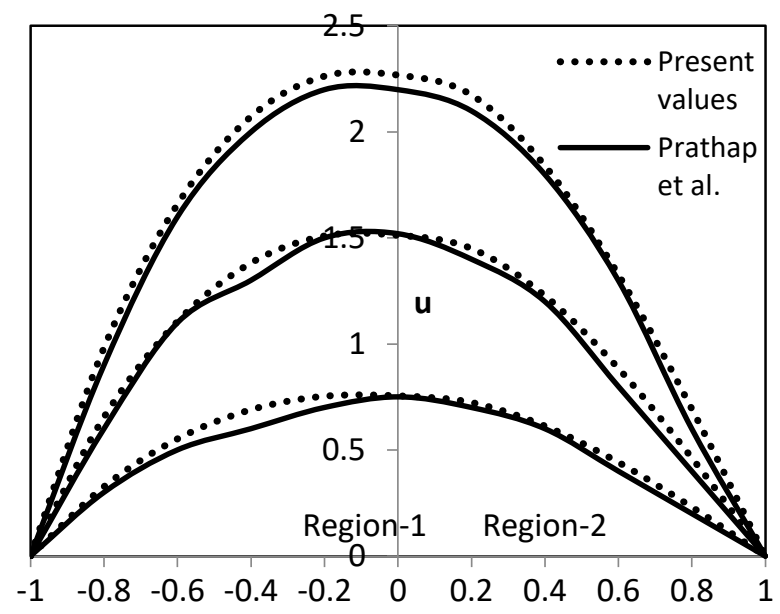

Figure2. Velocity profiles for $\mathrm{Gr}=5,15,20$ at $\mathrm{R}=1, \mathrm{~K}^{\prime}=1, \mathrm{~h}=1, \alpha=1, \mathrm{~m}=1, \mathrm{~b}=1$.

The curves in Figure 3 and Figure 4 illustrate the effect of the thermal Grashof number and mass Grashof number for the heat and mass transfer on the velocity and angular velocity (micro rotation) profiles. The Grashof number for heat transfer signifies the relative effect of the thermal buoyancy force to the viscous hydrodynamic force in the boundary layer. As expected, the velocity increases in both regions because of the enhancement of the thermal buoyancy force. Also, as the Gr increases the peak values for velocity are attained in the viscous region and clearly the velocity increases more rapidly in the viscous region than in the micropolar region due to the presence of micropolar molecules. The Grashof number for mass transfer defines the ratio of the species buoyancy force to the viscous hydrodynamic force. As expected, the fluid velocity increases and the peak value is more distinctive due to the increase in the species 
buoyancy force. The angular velocity plays a major role as we are considering the micropolar fluid in the first region. The effect of both the $\mathrm{Gr}$ and the Gcon angular velocity is depicted in curves 3(b), 4(b). It is seen that the angular velocity increases in line with the increase of the pertinent parameters in each case.
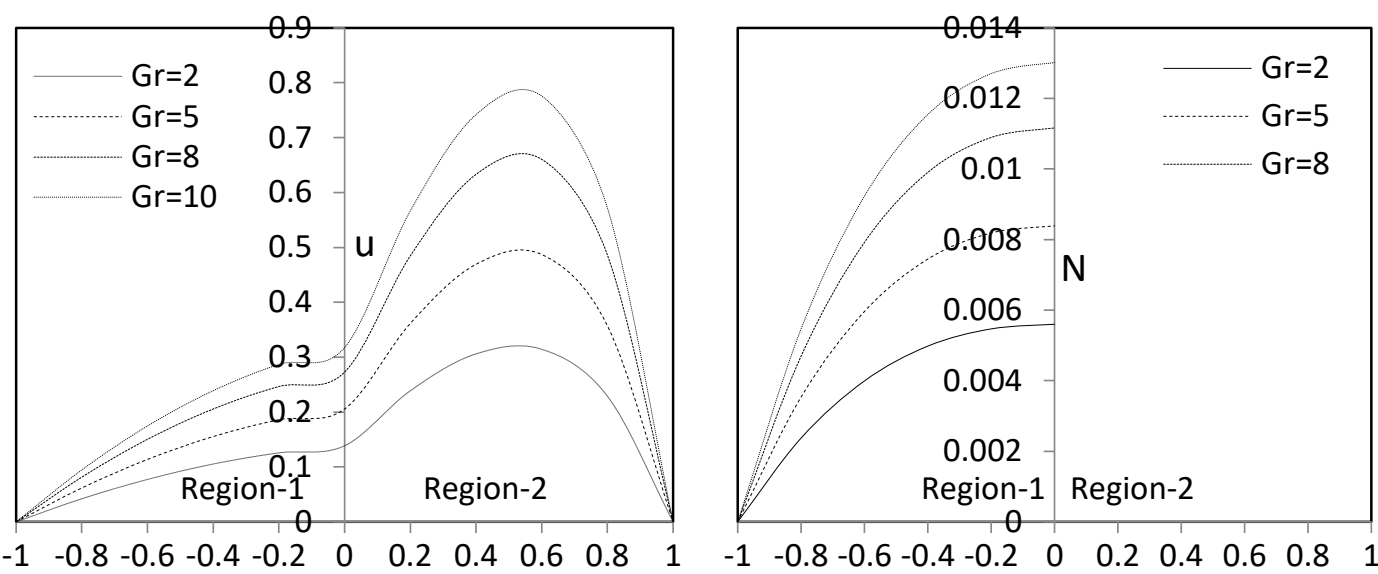

Figure 3. (a) Velocity profiles (b) Microrotation profiles for different values of Gr.
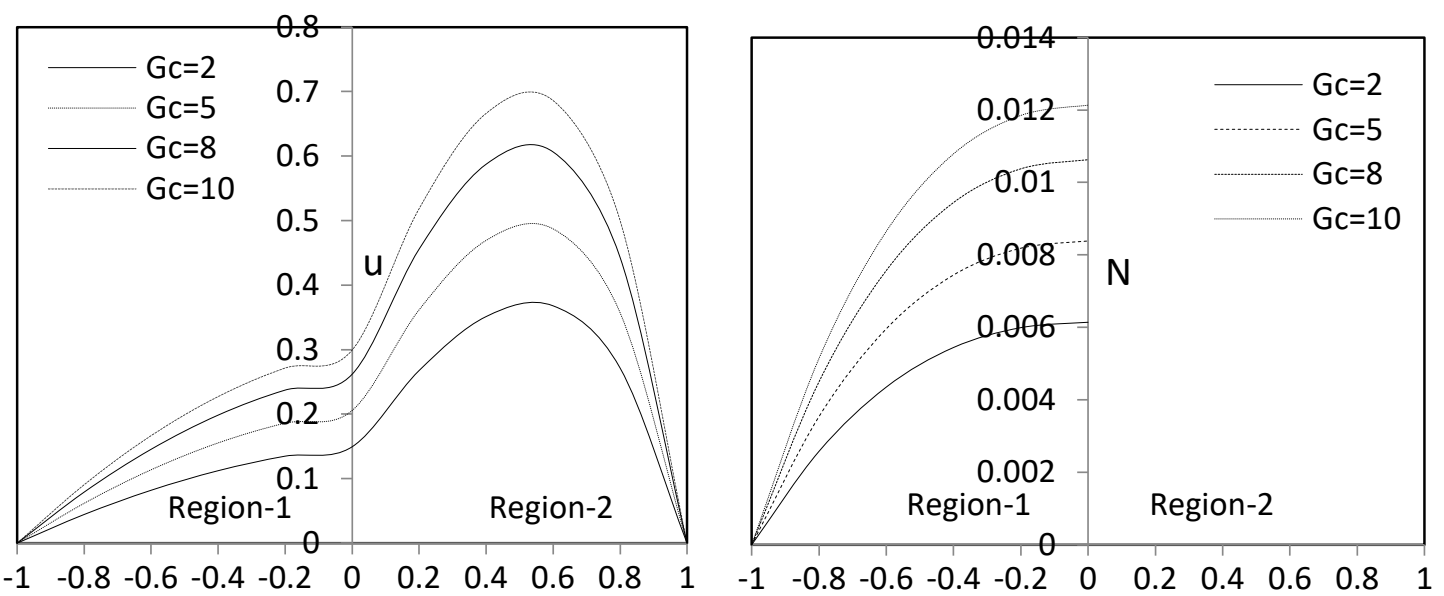

Figure 4. (a) Velocity profiles (b) Microrotation profiles for different values of Gc.

Figures 5 shows the influence of the Reynolds number on velocity and angular velocity. The Reynolds number is the ratio of inertial forces to viscous forces within a fluid which is subjected to a relative internal movement due to different fluid velocities. The increase of this number leads to a decay in velocity and in angular velocity. The effect of the magnetic field parameter on both velocities is described in Figure 6. It is interesting to note that the effect of the magnetic field is to decrease the value of the velocity profiles throughout the boundary layer. The effect of the magnetic field is more prominent in the viscous region, because the presence of the magnetic field in an electrically conducting fluid introduces a force called the Lorentz force, which acts against the flow. Figure 7 shows the effect of the material parameter on velocity and angular velocity. It is clear that both velocity and angular velocity increase with the increase of $K^{\prime}$, i.e. the fluid flow can be controlled by the dynamics of the material parameter which is used in the momentum 
and angular momentum equations of the micropolar region. When $K^{\prime}=0.03$, the fluid velocity attains more in both regions.
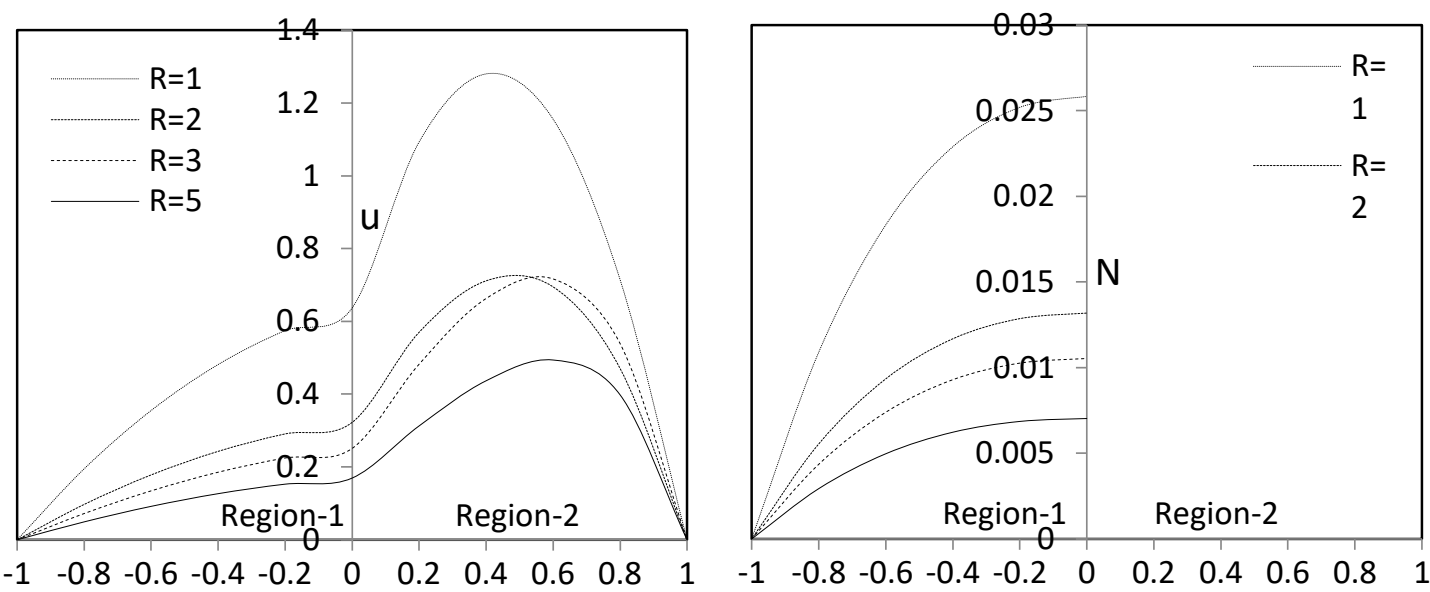

Figure 5. (a) Velocity profiles (b) Microrotation profiles for different values of R.
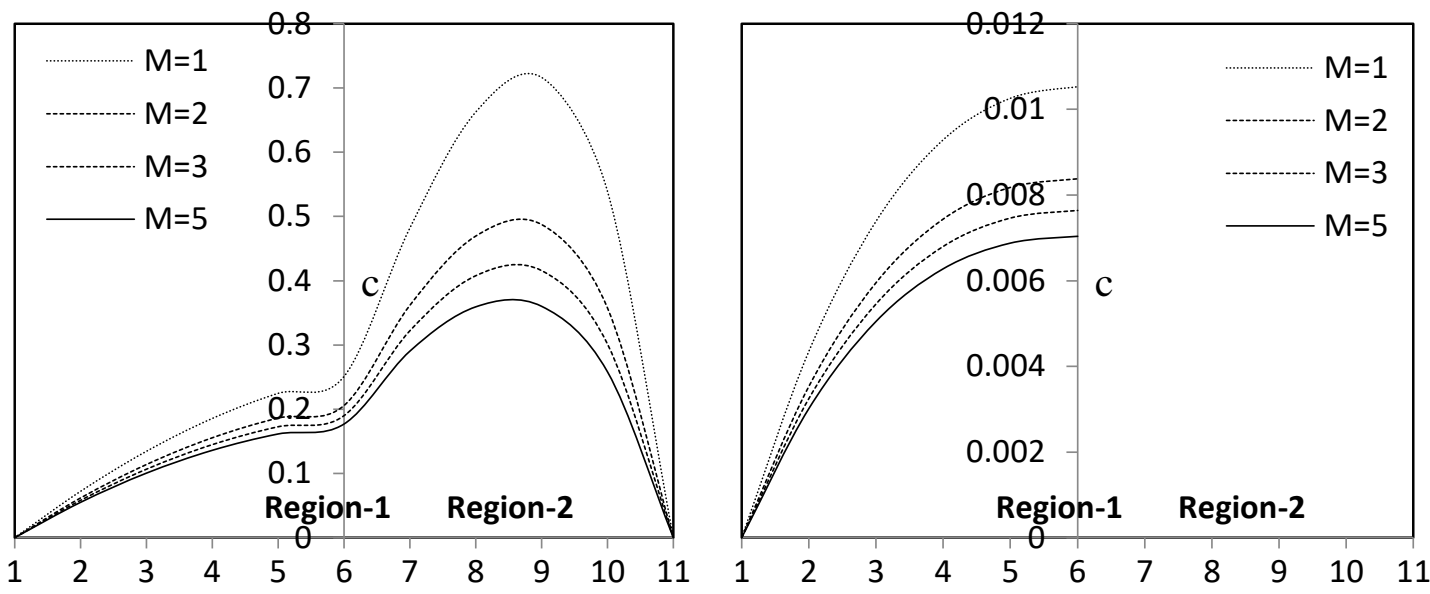

Figure 6. (a)Velocity profiles (b) Microrotation profiles for different values of M.
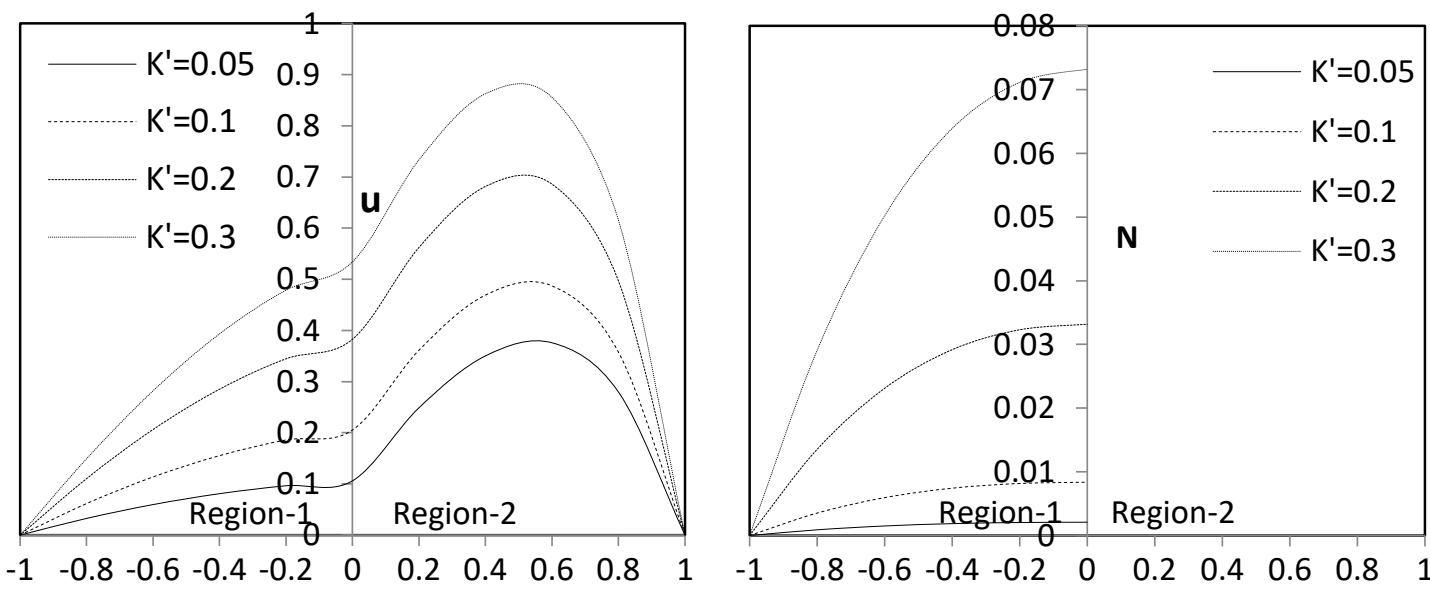

Figure 7. (a) Velocity profiles (b) Microrotation profiles for different values of K' 

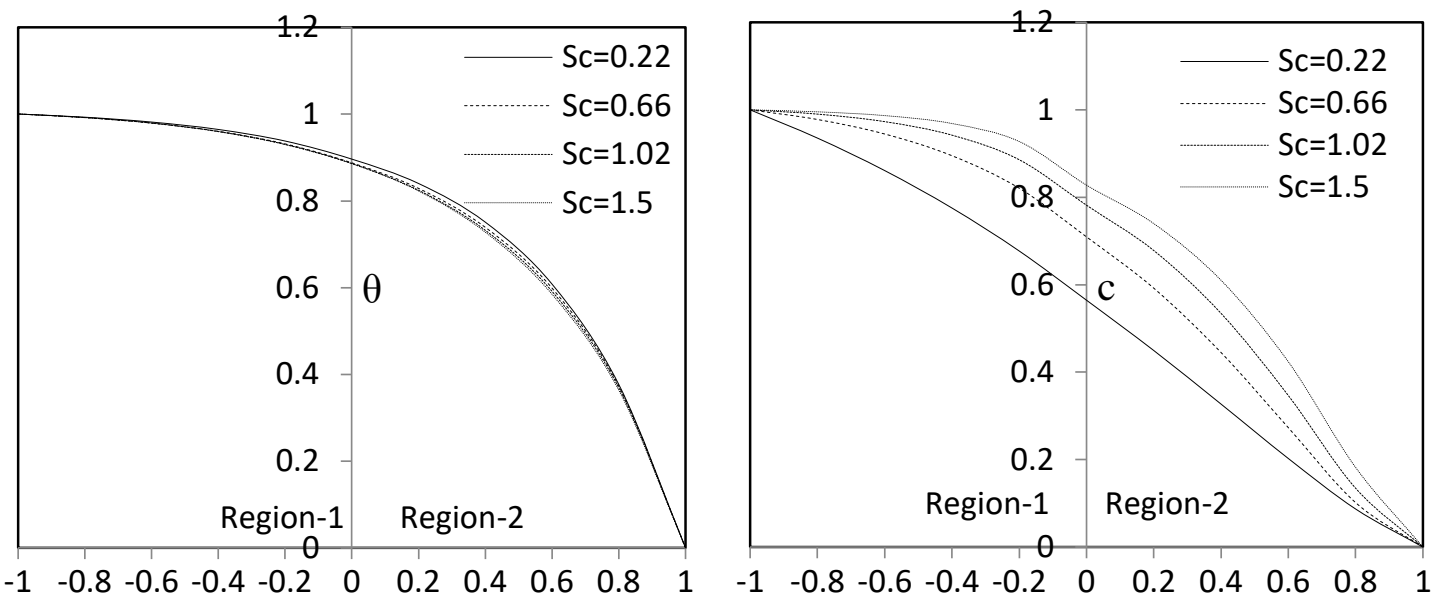

Figure 8. (a) Temperature profiles (b) Diffusion profiles for different values of Sc.
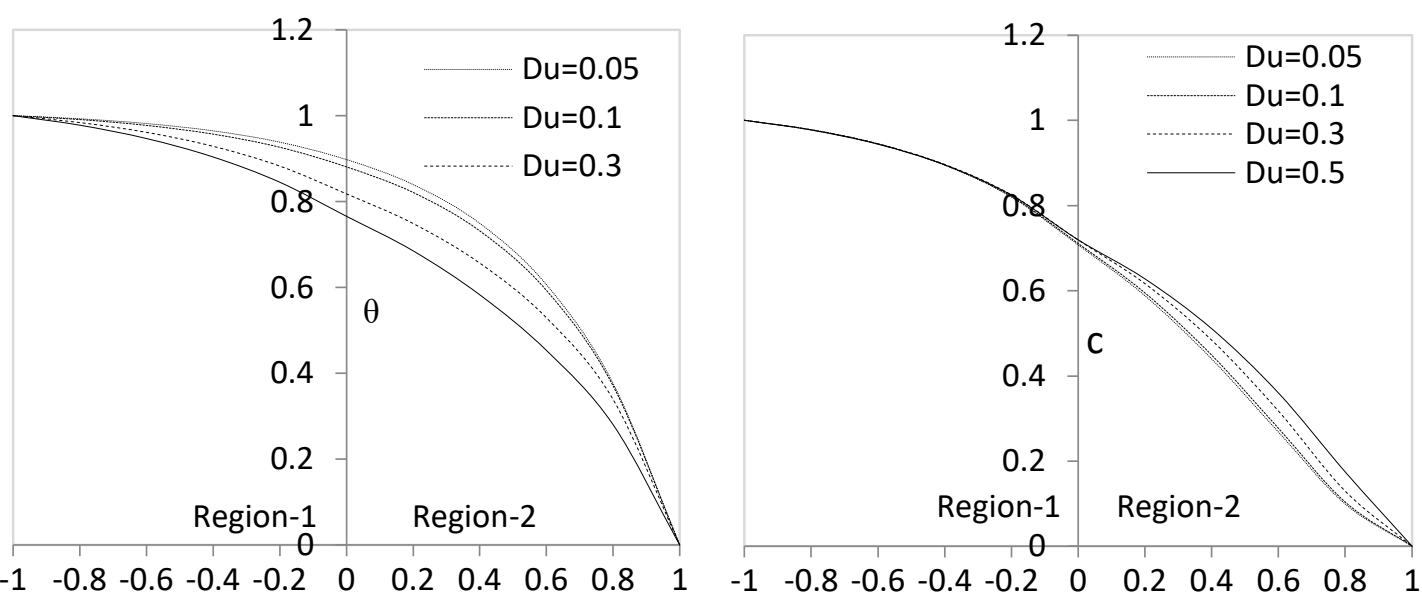

Figure 9. (a) Temperature profiles (b) Diffusion profiles for different values of Du.

The effects of the pertinent parameters of the energy and diffusion equations on the temperature and diffusion (concentration) profiles are presented from Figure 8 to Figure 12. The effect of the Schmidt number on the temperature and concentration profiles is shown in Figure 8. The Schmidt number embodies the ratio of the momentum to the mass diffusivity. The Schmidt number therefore quantifies the relative effectiveness of the momentum and mass transport by diffusion in the temperature and concentration (species). As the Schmidt number increases, the temperature decreases and the concentration increases. This behaviour is clear from both figures. The influence of the Dufour number $(\mathrm{Du})$ for different values on the temperature and diffusion profiles is plotted in Figure 9. The Dufour number indicates the contribution of the concentration gradients to the thermal energy flux in the flow. It is found that an increase in the Dufour number causes a drop in temperature throughout the region and rise in the species concentration. Figure 10 depicts the temperature and concentration profiles for different values of the Soret number ( $\mathrm{Sr}$ ). The Soret number defines the effect of the temperature gradients inducing significant mass diffusion effects. It is noticed that an increase in the Soret number results in an increase in the velocity and concentration within the boundary layer. It is noticed that an increase in the Soret number results in an increase in the temperature and decay in the species concentration. The effects of the Reynolds number 
is depicted from Figure 11, the effect of the Reynolds number on temperature and concentration shows that the increase of $\mathrm{R}$ leads to an increase in the temperature and species concentration. The influence of the viscous dissipation parameter i.e., the Eckert number on the temperature and concentration is shown in Figure 12. The Eckert number expresses the relationship between the kinetic energy in the flow and the enthalpy. It embodies the conversion of kinetic energy into internal energy by work done against the viscous fluid stresses. Greater viscous dissipative heat causes a rise in the temperature as well as in the species concentration.
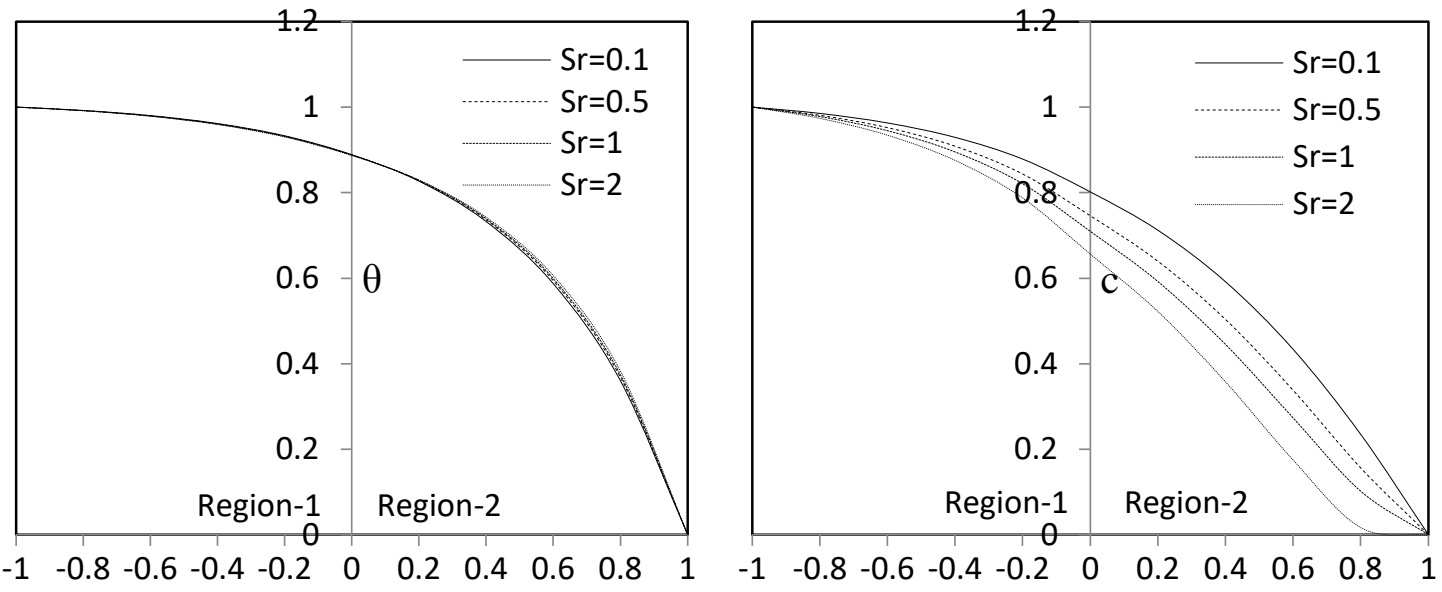

Figure 10. (a) Temperature profiles (b) Diffusion profiles for different values of Sr.


Figure 11.(a) Temperature profiles (b) Diffusion profiles for different values of $\mathrm{R}$.

Table 1 shows the Nusselt number (rate of heat transfer) and the Sherwood number (rate of mass transfer) values with the effects of the Reynolds number, Dufour number, Soret number, Schmidt number and Eckert numbers at both boundaries. From this table, it is observed that the Nusselt number decreases near the boundary at $y=-1$ and increases near the boundary $y=1$ in line with the increase of the Reynolds number and Eckert number. The rate of heat transfer increases near the left boundary and decreases near the right boundary for the increase of $\mathrm{Du}, \mathrm{Sr}, \mathrm{Sc}$. The Sherwood number decreases near both the plates with the effect of $\mathrm{R}$ and Ec and increases near both the plates with the variations of Du. Also it is noticed that the Sherwood number increases 
near the boundary at $y=-1$ and decreases at the boundary $y=1$ for variations of Sc, Ec and for the variations of $\mathrm{Sr}$, the Sherwood number increases near the left plate and decreases near the right plate.

Table 1. Nusselt number and Sherwood numbers.

\begin{tabular}{ccccccccc}
\hline $\mathrm{R}$ & $\mathrm{Du}$ & $\mathrm{Sr}$ & $\mathrm{Sc}$ & $\mathrm{Ec}$ & $\mathrm{Nu}-\mathrm{I}$ & $\mathrm{Nu}-\mathrm{II}$ & $\mathrm{Sh}-\mathrm{I}$ & Sh-II \\
\hline 1 & 0.08 & 0.1 & 0.66 & 0.001 & 0.225936 & 0.939699 & 0.282894 & 0.741633 \\
3 & 0.08 & 0.1 & 0.66 & 0.001 & 0.0330117 & 2.35331 & 0.0882108 & 0.180164 \\
3 & 0.08 & 0.1 & 0.66 & 0.001 & 0.0282603 & 2.35685 & 0.0881097 & 0.175324 \\
3 & 0.5 & 0.1 & 0.66 & 0.001 & 0.0512643 & 2.33058 & 0.0890344 & 0.207741 \\
3 & 0.08 & 0.08 & 0.66 & 0.001 & 0.0868553 & 1.55754 & 0.149199 & 0.862875 \\
3 & 0.08 & 0.13 & 0.66 & 0.001 & 0.0872974 & 1.61949 & 0.189429 & 0.399887 \\
3 & 0.08 & 0.1 & 0.22 & 0.001 & 0.0807822 & 1.60706 & 0.355558 & 0.522276 \\
3 & 0.08 & 0.1 & 1.5 & 0.001 & 0.0884291 & 1.49468 & 0.0440824 & 1.31943 \\
3 & 0.08 & 0.1 & 0.66 & 0.001 & 0.0870215 & 1.5817 & 0.165373 & 0.682477 \\
3 & 0.08 & 0.1 & 0.66 & 0.05 & 0.0820235 & 1.64615 & 0.165565 & 0.644296 \\
\hline
\end{tabular}
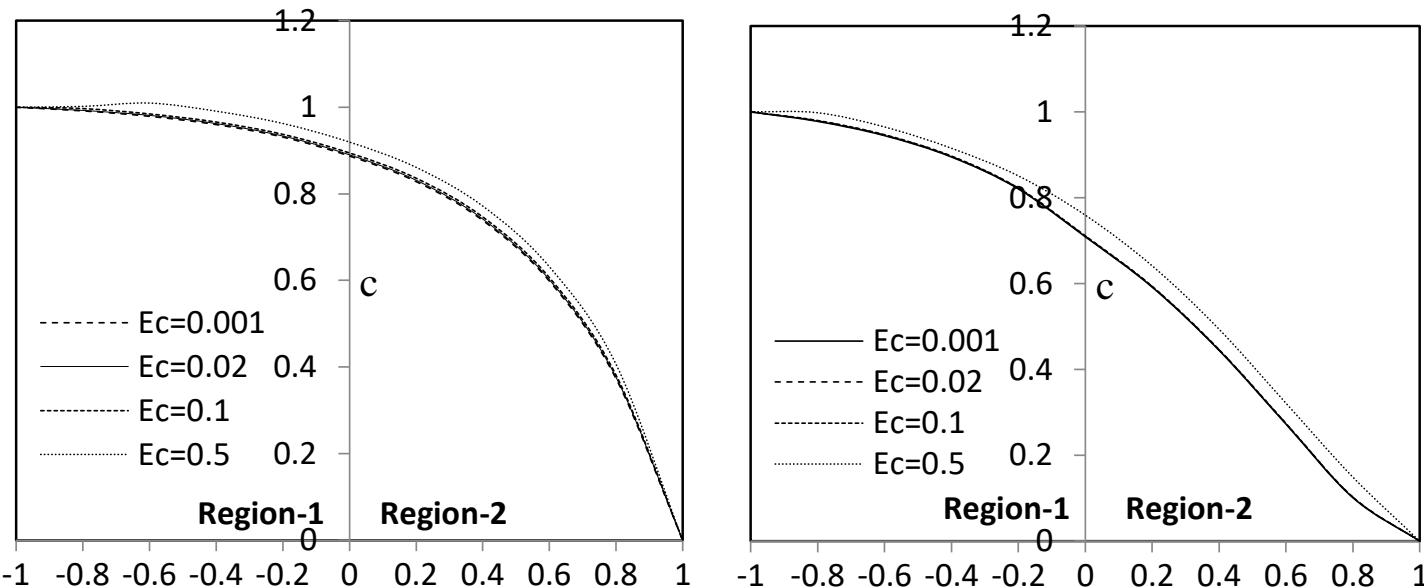

Figure 12. (a) Temperature profiles (b) Diffusion profiles for different values of Ec.

Table 2. Shear stress values

\begin{tabular}{cccccc}
\hline Gr & Gc & R & M & St-I & St-II \\
\hline 2 & 5 & 3 & 3 & -0.226663 & 1.5808 \\
5 & 5 & 3 & 3 & -0.332107 & 2.47969 \\
10 & 5 & 3 & 3 & -0.50786 & 3.97814 \\
5 & 2 & 3 & 3 & -0.238284 & 1.89073 \\
5 & 5 & 3 & 3 & -0.332107 & 2.47969 \\
5 & 10 & 3 & 3 & -0.48848 & 3.46125 \\
5 & 5 & 1 & 3 & -1.06105 & 4.16442 \\
5 & 5 & 2 & 3 & -0.524616 & 3.00459 \\
5 & 5 & 5 & 3 & -0.175332 & 1.84106 \\
5 & 5 & 3 & 1 & -0.298256 & 1.77122 \\
5 & 5 & 3 & 2 & -0.313686 & 2.07823 \\
5 & 5 & 3 & 5 & -0.383676 & 3.78302 \\
\hline
\end{tabular}


Table 2 shows the shear stress values for different values of Gr, Gc, R and M near both boundaries. From this table, it is observed that the shear stress decreases on the boundary at $y=-1$ and increases at the boundary $y=1$ with the increase of Gr, Gc and M. The magnitude of shearing is decreasing near both boundaries.

\section{CONCLUSIONS}

The thermo-diffusion and diffusion-thermo effect on convective heat and on the mass transfer of micropolar and viscous fluids in a vertical channel using finite element method is presented. It is concluded that the Galerkin finite element method is validated by comparing with the analytical method used by the previous authors. The diffusion effects are significant in both regions. The diffusion thermo effect enhances the temperature and the thermal diffusion reduces the temperature in both regions. The velocity, temperature and diffusion are lowered due to the presence of micropolar molecules. The diffusion thermo effect reduces the rate of heat transfer and enhances the rate of diffusion. The thermo diffusion effect enhances the rate of heat transfer and reduces the rate of diffusion.

\section{ACKNOWLEDGEMENTS}

Authors would like to sincerely thank the reviewers for their useful and constructive discussions and valuable suggestions which led to a definite improvement of the quality of the manuscript.

\section{REFERENCES}

[1] Malashetty M, Umavathi J, Kumar JP. Convective magnetohydrodynamic two fluid flow and heat transfer in an inclined channel. Heat and Mass Transfer. 2001;37:259-64.

[2] Malashetty M, Umavathi J, Prathap Kumar J. Two-fluid magnetoconvection flow in an inclined channel. Int J Trans Phenomena. 2001;3:73-84.

[3] Malashetty M, Leela V. Magnetohydrodynamic heat transfer in two phase flow. International Journal of Engineering Science. 1992;30:371-7.

[4] Muthuraj R, Srinivas S. Fully developed MHD flow of a micropolar and viscous fluids in a vertical porous space using HAM. Int J Appl Math Mech. 2010;6:5578.

[5] Kumar N, Gupta S. MHD free-convective flow of micropolar and Newtonian fluids through porous medium in a vertical channel. Meccanica. 2012;47:277-91.

[6] Srinivas J, Murthy JR. Flow of two immiscible couple stress fluids between two permeable beds. Journal of Applied Fluid Mechanics. 2016;9:501-7.

[7] Eringen AC, Suhubi E. Nonlinear theory of simple micro-elastic solids-I. International Journal of Engineering Science. 1964;2:189-203.

[8] Lukaszewicz G. Micropolar fluids: theory and applications: Springer Science \& Business Media; 1999.

[9] Eringen AC. Microcontinuum field theories: II. Fluent media: Springer Science \& Business Media; 2001.

[10] Kumar JP, Umavathi JC, Chamkha AJ, Pop I. Fully-developed free-convective flow of micropolar and viscous fluids in a vertical channel. Applied Mathematical Modelling. 2010;34:1175-86. 
[11] Srinivas G, Reddy B. Finite element analysis of free convection flow with MHD micropolar and viscous fluids in a vertical channel with dissipative effects. Journal of Naval Architecture and Marine Engineering. 2011;8:59-69.

[12] Kurnia JC, Sasmito AP. Heat transfer performance of non-circular coiled tubes Research summary, challenges and directions. International Journal of Automotive and Mechanical Engineering. 2016;13:3710-27.

[13] Chala GT, A. Sulaiman S, Japper-Jaafar A, Wan Abdullah WAK. Investigation of convective heat transfer coefficient and initial temperature of waxy crude oil on the formation of voids. International Journal of Automotive and Mechanical Engineering. 2016;13:3754-62.

[14] Sansaniwal SK, Kumar M. Analysis of ginger drying inside a natural convection indirect solar dryer: An experimental study. Journal of Mechanical Engineering and Sciences. 2015;9:1671-85.

[15] Nawi MRM, Mamat AMI, Ismail H. Numerical heat transfer analysis of waste heat exchanger for exhaust gas energy recovery. Journal of Mechanical Engineering and Sciences. 2015;8:1498-506.

[16] Kumar M, Bhutani V, Khatak P. Research progresses and future directions on pool boiling heat transfer. Journal of Mechanical Engineering and Sciences. 2015;9:1538-55.

[17] Bég O, Bhargava R, Rawat S, Kahya E. Numerical study of micropolar convective heat and mass transfer in a non-Darcy porous regime with Soret and Dufour diffusion effects. Emirates Journal for Engineering Research,. 2008;13:51-66.

[18] Lohrasbi J, Sahai V. Magnetohydrodynamic heat transfer in two-phase flow between parallel plates. Applied Scientific Research. 1988;45:53-66.

[19] Sheri SR, Suram AK, Modulgua P. Heat and mass transfer effects on mhd natural convection flow past an infinite inclined plate with ramped temperature. Journal of the Korean Society for Industrial and Applied Mathematics. 2016;20:355-74.

[20] Zivojin SM, Nikodijevic DD, Blagojevic BD, Savic SR. MHD flow and heat transfer of two immiscible fluids between moving plates. Transactions of the Canadian Society for Mechanical Engineering. 2010;34:351-72.

[21] Narayana PS, Ramireddy G, Venkataramana S. Hall current effects on free convection MHD flow past a porous plate. International Journal of Automotive and Mechanical Engineering. 2011; 3:350-63.

[22] Koriko OK, Oreyeni T, Omowaye AJ, Animasaun IL. Homotopy analysis of MHD free convective micropolar fluid flow along a vertical surface embedded in non-darcian thermally-stratified medium. Open Journal of Fluid Dynamics. 2016;6:198-221.

[23] Ariman T, Turk M, Sylvester N. Microcontinuum fluid mechanics-a review. International Journal of Engineering Science. 1973;11:905-30.

[24] Reddy JN. An introduction to the finite element method: McGraw-Hill New York; 1993.

[25] Zienkiewicz OC, Taylor RL, Zienkiewicz OC, Taylor RL. The finite element method: McGraw-hill London; 1977.

[26] Sedighi M, Dardashti BN. Finite element analysis of heat transfer in multi-layer cooking pots with emphasis on layer number. International Journal of Automotive and Mechanical Engineering. 2015; 11:2253-61. 